\title{
Spatial disinhibition of orientation analyzers
}

\author{
N. R. LONG and J. G. M. SCHEIRLINCK \\ Massey University, Palmerston North, New Zealand
}

\begin{abstract}
Following the successive presentation of two masking gratings (M1 and M2), subjects were required to detect the presence or absence of a single vertical line (TS). When the orientations of the two masks were optimal, M1 was able to reduce the masking effect of M2 on the TS. For a vertical TS, disinhibition was maximal when the orientations of M1 and M2 were similar and was minimal when the orientational difference was greater than $15 \mathrm{deg}$. It is suggested that the spatial selectivity of the disinhibition function reflects the activity of neurons tuned to orientation, and that the disinhibition masking paradigm may be a useful psychophysical technique to measure tuning functions of other feature detectors.
\end{abstract}

When a visual target stimulus (TS) is presented within close temporal and/or spatial contiguity with a masking stimulus, the masking stimulus is able to reduce the detectability of the TS. If a second mask is introduced into the masking sequence, the introduced mask is able to disinhibit (reduce the masking effect of) the first mask (Dember \& Purcell, 1968; Dember, Schwartz, \& Kocak, 1978; Kristofferson, Galloway, \& Hanson, 1979; Robinson, 1966). In these studies the TS was often a single uppercase letter, while the first mask (M1) and the second mask (M2) were a disk and either a larger disk or ring, respectively. Maximum disinhibition has been reported at stimulus onset asynchronies (SOAs) of $116 \mathrm{msec}$ (TS-M2) and $35 \mathrm{msec}$ (TS-M1) (Kristofferson et al., 1979). In the experiments that have reported disinhibition, the effect is critically dependent upon the SOAs, the masking ability and the energy levels within the masks (Breitmeyer, 1978; Bryon \& Banks, 1980; Long \& Gribben, 1971). Other experiments that have varied stimulus size, configuration, luminance, and the forced-choice procedure have failed to report disinhibition (Barry \& Dick, 1972; Prager \& Matteson, 1978; Schurman \& Eriksen, 1969). For disinhibition to occur, M1 must exert a moderate masking effect on TS and, provided that the SOAs are sufficient to prevent summation between the masks, then M2 will disinhibit Ml (Long \& Gribben, 1971).

Many theoretical explanations have been offered to account for masking and disinhibition (e.g., interruption, integration, and overtake), but theories based on neurophysiological properties of feature detecting neurons are obviously relevant when the TS and MS temporal and spatial relationships determine masking effects (Breitmeyer, 1980; Breitmeyer \& Ganz, 1976; Weisstein, Ozog, \& Szoc,

Requests for reprints should be sent to N. R. Long, Department of Psychology, Massey University, Palmerston North, New Zealand.
1975). The basic assumption of a neural theory of disinhibition is that the activity of a neuron can be modulated by prior activity and/or by the activity of neighboring units. Following prolonged optimal stimulation, a neuron undergoes a period of postexcitatory suppression. If a near-threshold TS is presented during this period, it must be presented at a higher energy level for detection. Also, interchannel inhibition may modify a neuron's response, but only to the extent that the channels are responsive to the same or overlapping spatial dimensions in the stimuli. This latter explanation has been offered to account for the occurrence of disinhibition in orientation illusion (Blakemore, Carpenter, \& Georgeson, 1970; O'Toole, 1979), aftereffect (Magnussen \& Kurtenbach, 1980), and masking (Wenderoth \& Tyler, 1979).

Barry and Dick (1972) and Purcell and Dember (1968) have suggested that disinhibition in Robinson's (1966) paradigm may arise as successive brightness contrast or brightness reversal. Implicit in this explanation is that disinhibition arises within populations of neurons that are maximally responsive to luminance properties of $\mathrm{M} 1$ and M2. More powerful support for a feature detection explanation is evidenced by the spatial selectivity of motion disinhibition observed when the direction of a moving grating is varied (Levinson \& Sekuler, 1975). Using the direction-specific properties of motion detectors, Levinson and Sekuler (1975) determined the threshold of a rightward moving test grating (i.e., for this moving grating, the inhibitory component moved leftwards). Subsequent preadaptation to a leftward moving grating disinhibited the inhibitory component of the rightward moving grating with a subsequent reduction in the threshold of the test grating. Similar spatial restrictions of disinhibition when lines and bars have been utilized have been reported (Breitmeyer, 1978; Rentschler \& Hilz, 1976). Breitmeyer reported that the masking effect of briefly presented bars on the detection of a vernier 
target was reduced when two disinhibiting bars were presented, and that the reduction was dependent on the spatial configuration of the stimuli. For example, when the disinhibiting bars were laterally separated by 20 minarc from the mask, there was a substantial increase in masking. These spatial restrictions on the magnitude of disinhibition suggest that the effect is likely to occur at a high level in the visual system.

Several studies have attempted to isolate the locus of the disinhibitory effect by utilizing dichoptic masking (Robinson, 1968; Turvey, 1973). However, interpretation of these results is difficult since identification of the locus of the interaction on the basis of the presence or absence of interocular effects is insufficient (see Long, 1979). If disinhibition arises as the direct result of an interchannel inhibitory process within feature detecting neurons, then the effect should be spatially restricted to the known receptive field properties (e.g., tuning ranges) of the neurons. The importance of this additional requirement is that it implies a central locus for the effect, as there is strong evidence that suggests that spatial selectivity is a major property of neurons in the visual cortex (Hubel \& Wiesel, 1968; Maffei \& Fiorentini, 1973; Poggio \& Fischer, 1977).

The spatial limitation of the masking functions obtained in feature masking has been attributed to the inhibitory interaction of neural units (Breitmeyer, 1980; Weisstein et al., 1975). Furthermore, the selectivity observed in the masking functions is spatially restricted to the stimulus features that have similar spatial values (Blakemore \& Hague, 1972; Blakemore, Nachmias, \& Sutton, 1970; Over, Broerse, \& Crassini, 1972).

Blakemore et al. (1970) and O'Toole (1979) have demonstrated orientation disinhibition by observing the apparent contraction or expansion of acute angles when a third disinhibitory line is introduced. In both of these experiments, the maximum disinhibitory effect occurred when the difference between the masking and disinhibiting orientations was $10-20 \mathrm{deg}$. In the present study, a neural explanation of visual masking is offered to account for spatial disinhibition observed in orientation illusion and masking. Orientational selectivity has been demonstrated directly from microelectrode recording from neurons in the monkey cortex (Hubel \& Wiesel, 1968), visually evoked cortical potentials from humans (Campbell \& Maffei, 1970), and from other human psychophysical data (Gilinsky \& Mayo, 1971; Over et al., 1972). There are theoretical problems associated with the linking of electrophysiological data based on the activity of single neurons and psychophysical data (Sekuler, 1974; Wenderoth \& Latimer, 1979). However, the aim is not to infer equivalence of neural functioning, but to offer support for the psychophysical data when the further restraint of physiological data is considered (Breitmeyer, 1980). In this study, it is hypothesized that the detectability of a vertical target line will vary as a function of the orientation of the masking gratings. Specifically, it is proposed that orientation disinhibition should occur only when the orientations of M1 and M2 maximally mask each other.

\section{METHOD}

Subjects

Three undergraduate students with normal or corrected-tonormal vision served as subjects.

\section{Procedure}

A forward masking-disinhibition paradigm was used. Following the presentation of a centrally located fixation spot $(10 \mathrm{sec})$, the stimulus sequence commenced with M1 presented for $150 \mathrm{msec}$. Interstimulus 1 (ISI1) for $1 \mathrm{msec}, \mathrm{M} 2$ for $150 \mathrm{msec}$, ISI2 for 10 msec, and then the TS (or homogeneous blank field on catch presentations). The duration of the TS was $4 \mathrm{msec}$ and was the duration at which the subjects could correctly detect the TS at $75 \%$ accuracy and when a homogeneous MS of $26.0 \mathrm{~cd} / \mathrm{m}^{2}$ was displayed beforehand. A homogeneous rather than a grating MS was used to ensure that any subsequent change in the detectability of the TS was due to the presence of oriented features within the mask. The stimuli were aligned so that the features impinged on the same retinal area when displayed in separate channels of a Scientific Prototype tachistoscope.

The TS was a single black vertical line, $7 \mathrm{deg} 8 \mathrm{~min}$ long and $9 \mathrm{~min}$ wide, while the square-wave gratings utilized for $\mathrm{M} 1$ and M2 subtended $7 \mathrm{deg} 8 \mathrm{~min}$, and each line of the grating subtended 9 min wide. All MS displays were circular and could be rotated. Four orientation values of $M 1$ and $M 2$ were used $(0,7,15$, and $30 \mathrm{deg}$ from vertical). The space average luminance of the TS (or catch stimulus) was maintained at $29.5 \mathrm{~cd} / \mathrm{m}^{2}$ and was $26.0 \mathrm{~cd} / \mathrm{m}^{2}$ for $\mathrm{M} 1$ and $\mathrm{M} 2$.

In each session, the subject was given $30 \mathrm{~min}$ of practice in reporting the presence of the TS when one MS was used. During the practice session, the subject's TS threshold was determined, and this value was subsequently used throughout the experiment. All orientation combinations of $\mathrm{M} 1$ and $\mathrm{M} 2$ were randomized into 16 blocks and were balanced in accord with a Latin square so that all subjects received all combinations in different orders. Four further blocks of all M1 orientations without the presence of M2 (i.e., normal forward masking condition) were also included to serve as controls against which disinhibition could be measured. Each block consisted of 12 trials made up of six trials on which the TS was actually present, randomly interspersed with six catch trials. On the six target trials, the single vertical line was presented, while on the catch trials, a homogeneous field of equal luminance was shown. The subject's task was to report whether the TS was present or absent (i.e., to differentiate between the target and catch trials). Testing was conducted in a dark room, with each subject triggering the tachistoscope. There was approximately $10 \mathrm{sec}$ between trials and 1 min between blocks.

\section{RESULTS}

The percent correct detection of the TS was calculated for each subject under all factorial combinations of M1 and M2, and was the accuracy with which each subject could correctly detect the presence or absence of the TS. An anlysis of variance was performed on these data. The percent detection varied significantly as a function of the orientation 


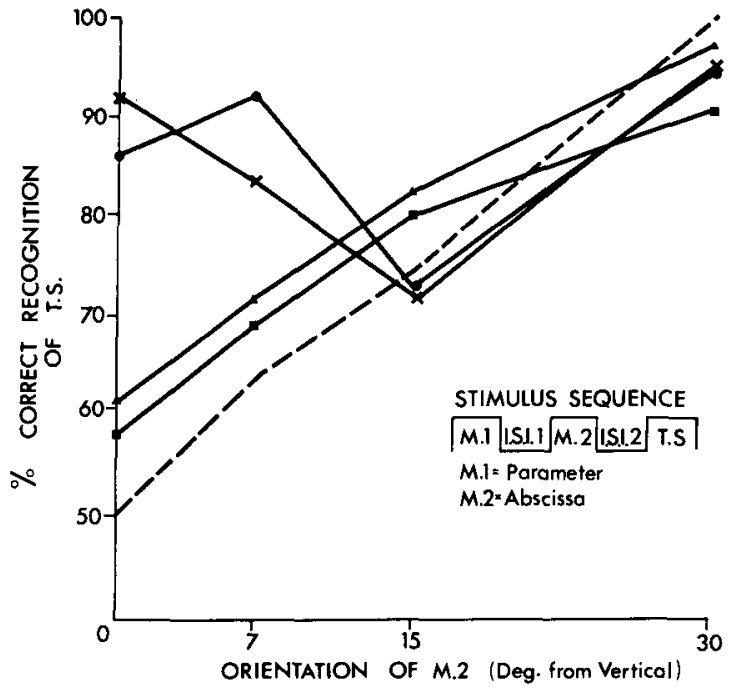

Figure 1. Percentage correct detection of TS as a function of the orientations of M1 and M2. Parameter is M1 orientation $(X \longrightarrow X, 0$ deg; $\bullet-\bullet, 7$ deg; $\Lambda \longrightarrow \triangle, 15$ deg; -, $30 \mathrm{deg})$ and forward masking control ( ---$)$.

of $\mathrm{M} 2[\mathrm{~F}(3,6)=18.65, \mathrm{p}<.01]$, while the orientation of M1 did not exert a significant influence $[F(3,6)=4.49, p>.05]$. The two-way interaction between these variables was significant $[F(9,18)=$ $7.48, \mathrm{p}<.01]$ and is shown in Figure 1.

In order to directly compare the disinhibition and the forward masking control condition, the percent correct detection scores from the disinhibition data were pooled over M1 orientations and subjected to a further analysis of variance. While the orientation of M2 exerted a significant effect on the percent correct detection of the TS $[F(3,6)=20.46$, p $<$ $.01]$, there was no significant difference between the disinhibition and forward masking control conditions $[F(1,2)=11.46, p>.05]$. The two-way interaction between these variables was significant $[\mathrm{F}(3,6)=106.16, \mathrm{p}<.01]$. Multiple comparisons of means using the Newman-Keuls method of comparison revealed that the introduction of $\mathrm{M} 1$ prior to M2 significantly reduced the masking effect of $\mathrm{M} 2$, provided the orientation of $\mathrm{M} 2$ was either 0 or $7 \mathrm{deg}$. Inspection of Figure 1 showed that disinhibition occurred when both M1 and M2 were 0 or $7 \mathrm{deg}$. If $\mathrm{M} 2$ was 15 or $30 \mathrm{deg}$, then the introduction of a 0 - or 7-deg M1 had little effect, as the function was similar to the forward masking control condition. Similarly, the functions obtained by M1s of 15 and $30 \mathrm{deg}$ at all $\mathrm{M} 2$ orientations resembled the forward masking control condition. Generally, it appears that if disinhibition of vertical TS is to occur, then M1 and M2 must be processed by overlapping neural channels that are tuned to orientation values less than 15 deg to vertical.

\section{DISCUSSION}

The results of the present experiment give considerable support to the contention that the human visual system responds selectively to narrow ranges of orientation information. Following the presentation of masking stimuli containing orientation information, disinhibition occurred when M1 and M2 were similar in orientation, and disinhibition declined as the orientation difference between the masks increased. Orientation disinhibition was highly specific in that disinhibition failed to occur if the orientation of the masks differed by $15 \mathrm{deg}$.

The findings of this experiment are specific to a TS of $0 \mathrm{deg}$, since there is considerable evidence that suggests that oblique orientations are signaled by channels that are less specifically tuned and less represented within the visual system than vertical and horizontal orientations (Appelle, 1972; Matin \& Drivas, 1979). In order to predict the likelihood of orientation disinhibition between masks of various orientations, the different sensitivities of the visual system to oblique orientations must be taken into account. The current results, which suggest that M1 orientations of greater than 15 deg cannot disinhibit equal energy M2s of lesser orientations, are in partial agreement with those of Wenderoth and Tyler (1979), who reported that, in a simultaneous masking paradigm, a 0 -deg (i.e., vertical) grating could also be disinhibited by orientations of 75 and $90 \mathrm{deg}$ (i.e., approximately horizontal). With the exception of these latter results, the orientation over which disinhibition resulted is extremely similar to the orientation tuning ranges -isolated by other psychophysical techniques (Campbell \& Maffei, 1970; Fidell, 1972; Gilinsky \& Mayo, 1971; O’Toole, 1979; Over et al., 1972). The present data support the suggestion that disinhibition may arise by lateral inhibition in populations of feature detecting neurons. Furthermore, the orientational selectivity of the effect suggests that the disinhibition paradigm could be added to other psychophysical techniques as a further method to study feature detection in the human visual system.

The magnitude of disinhibition at optimal orientation values between $\mathbf{M} 1$ and $M 2$ is relatively large, and this finds support with the strong recovery effects reported by Dember et al. (1978), Kristofferson et al. (1979), and Wenderoth and Tyler (1979). The present experiment differs in several aspects from previous studies since forward disinhibition has been used, whereas backward disinhibition paradigms have almost universally been utilized in the past. The forward masking paradigm is utilized to maximize the masking effect of M1 on M2 (Wenderoth \& Tyler, 1979). Surprisingly, at the short ISI1 used in the present experiment, 
summation between the masks of identical orientation did not occur, but rather the neural activity generated by $\mathrm{M} 1$ reduced the masking effect of $\mathrm{M} 2$.

Two major theoretical accounts have been offered for disinhibition. Kristofferson et al. (1979) have proposed a recognition model based on the probability of detecting a target when the masking effect of the second mask on the target is zero. While this explanation is adequate for evaluating the detection likelihoods of combinations of stimuli, it has difficulty in accounting for the orientational selectivity obtained in the current data. Dember et al. (1978) have utilized Breitmeyer and Ganz (1976) and Weisstein et al.'s (1975) models to explain masking in terms of interchannel inhibition. In these models, sustained channels respond slowly to high spatial frequencies, and transient channels respond rapidly to low frequencies. Backward masking results from the transient units stimulated by the mask's overtaking and inhibiting the sustained units stimulated by the target. While this mechanism could account for backward masking, it had difficulty in explaining forward masking. This difficulty has been overcome by the proposal that sustained channels are also capable of inhibiting transient channels (Breitmeyer, 1980). Since the present experiment utilized stimuli with identical spatial frequencies, the results can be accounted for by this theory if it is assumed that the transient channels activated by the masks are processed more rapidly than the sustained channels.

In conclusion, a brief comment should be made on the absence of interocular disinhibition (Robinson, 1968). Long (1979) has argued that the presence or absence of interocular transfer is insufficient evidence on its own to indicate that the locus of ocular interaction is central or peripheral. In order to determine a central locus, spatial selectivity of the interaction must also be demonstrated. The failure of Robinson (1968) to demonstrate disinhibition suggests that brightness detectors are not binocularly driven or else that the effect is processed at a low level within the visual system. The present data suggest that orientation disinhibition may be a high level effect, and therefore interocular disinhibition for orientation analyzers should be able to be demonstrated. Microelectrode data support this contention, since a large number of orientation specific cells in the monkey's visual cortex are binocularly driven (Hubel \& Wiesel, 1968).

\section{REFERENCES}

Appelle, S. Perception and discrimination as a function of stimulus orientation: The "oblique effect" in man and animals. Psychological Bulletin, 1972, 78, 266-278.

BARRY, S. H., \& Dick, A. O. On the "recovery" of masked targets. Perception \& Psychophysics, 1972, 12, 117-120.

Blakemore, C., Carpenter, R. H. S., \& Georgeson, M. A. Lateral inhibition between orientation detectors in the human visual system. Nature, 1970, 228, 37-39.
Blakemore, C. B., \& Hague, B. Evidence for disparity detecting neurones in the human visual system. Journal of Physiology, $1972,225,437-455$.

Blakemore, C. B., Nachmias, J., \& Sutton, P. The perceived spatial frequency shift: Evidence for frequency-specific neurones in the human brain. Journal of Physiology, 1970, 210, 727-750.

Breitmeyer, B. G. Disinhibition in metacontrast masking of vernier acuity targets: Sustained channels inhibit transient channels. Vision Research, 1978, 18, 1401-1408.

Breitmeyer, B. G. Unmasking visual masking: A look at the "Why" behind the veil of "How." Psychological Bulletin, $1980,87,52-69$.

BREITMEYER, B. G., \& GANZ, L. Implications of sustained and transient channels for theories of visual pattern masking, saccadic suppression, and information processing. Psychological Review, $1976,83,1-36$.

Bryon, D., \& Banks, W. P. Pattern stimuli in disinhibition and backward masking. Bulletin of the Psychonomic Society, 1980, 15, 105-108.

Campbell, F. W., \& Maffei, L. Electrophysiological evidence for the existence of orientation and size detectors in the human visual system. Journal of Physiology, 1970, 207, 635-652.

Dember, W. N., \& Purcell, D. G. Recovery of masked visual targets by inhibition of the masking stimulus. Science, 1968, 157, 1335-1336.

Dember, W. N., Schwartz, M., \& Kocak, M. Substantial recovery of a masked visual target and its theoretical interpretation. Bulletin of the Psychonomic Society, 1978, 11, 285-287.

FideLl, L. S. Orientation specificity in chromatic adaptation of human edge detectors. Perception \& Psychophysics, 1970, 8, 235-237.

Gilinsky, A. S., \& Mayo, T. H. Inhibitory effects of orientational adaptation. Journal of the Optical Society of America, $1971,12,1710-1714$.

Huber, D. H., \& Wiesel, T. N. Receptive fields and functional architecture of the monkey striate cortex. Journal of Physiology, $1968,195,215-243$.

Kristofferson, A. B., Galloway, J., \& Hanson, R. G. Complete recovery of a masked visual target. Bulletin of the Psychonomic Society, 1979, 13, 5-6.

Levinson, E., \& SekUler, R. W. Inhibition and disinhibition of direction specific mechanisms in the human visual system. Nature, 1975, 254, 692-694.

Long, G. M. The dichoptic viewing paradigm: Do the eyes have it? Psychological Bulletin, 1979, 86, 391-403.

Long, N. R., \& GribBEN, J. A. The recovery of a visually masked target. Perception \& Psychophysics, 1971, 10, 197-200.

Maffe1, L., \& Fiorentini, A. The visual cortex as a spatial frequency analyzer. Vision Research, 1973, 13, 1255-1267.

Magnussen, S., \& Kurtenbach, W. Adapting to two orientations: Disinhibition in a visual aftereffect. Science, 1980, 207, 908-909.

Matin, E., \& Drivas, A. Acuity for orientation measured with a sequential recognition task and signal detection methods. Perception \& Psychophysics, 1979, 25, 161-168.

$O$ 'Toole, B. I. The tilt illusion: Length and luminance changes of induction line and third (disinhibiting) line. Perception \& Psychophysics, 1979, 25, 487-496.

Over, R., Broerse, J., \& Crassini, B. Orientation illusion and masking in central and peripheral vision. Journal of Experimental Psychology, 1972, 96, 25-31.

Poggio, G. H., \& Fischer, B. Binocular interaction and depth sensitivity in striate and prestriate cortex of behaving Rhesus monkey, Journal of Neurophysiology, 1977, 40, 1392-1405.

Prager, T. C., \& Matteson, H. H. Paracontrast and disinhibition. Bulletin of the Psychonomic Society, 1978, 12, 365-368.

Purcell, D. G., \& Dember, W. The relation of phenomenal brightness reversal and re-reversal to backward masking and recovery. Perception \& Psychophysics, 1968, 3, 290-292.

Rentschler, I., \& Hilz, R. Evidence for disinhibition in line detectors. Vision Research, 1976, 16, 1299-1302. 
Robinson, D. N. Disinhibition of visually masked stimuli. Science, 1966, 154, 157-158.

Robinson, D. N. Visual disinhibition with binocular and interocular presentation. Journal of the Optical Society of America, 1968, 58, 254-257.

Schurman, D. L., \& Eriksen, C. W. Summation and interaction of successive masking stimuli in visual perception. American Journal of Psychology, 1969, 82, 320-332.

Sekuler, R. W. Spatial vision. Annual Review of Psychology, 1974, 25, 194-232.

TURVEY, M. T. On peripheral and central processes in vision: Inferences from an information-processing analysis of masking with patterned stimuli. Psychological Review, 1973, 80, 1-52.

Weisstein, N., Ozog, G., \& Szoc, R. A comparison and elab- oration of two models of metacontrast. Psychological Review, 1975, 82, 325-343.

Wenderoth, P., \& Latimer, C. On the relationship between the psychology of visual perceptions and the neurophysiology of vision. In J. P. Sutcliffe (Ed.), Conceptual analysis and method in psychology: Essays in honour of $W . M$. O'Neil. Sydney: Sydney University Press, 1979.

WENDeroth, P., \& TyLER, T. The role of apparent motion cues in orientation masking. Perception \& Psychophysics, 1979, 25, 413-418.

(Received for publication December 20, 1980; accepted January 9, 1981.) 